\title{
Asymptotic behaviour of a transport equation
}

\author{
by RYsZard Rudnicki (Katowice)
}

\begin{abstract}
We study the asymptotic behaviour of the semigroup of Markov operators generated by the equation $u_{t}+b u_{x}+c u=a \int_{0}^{a x} u(t, a x-y) \mu(d y)$. We prove that for $a>1$ this semigroup is asymptotically stable. We show that for $a \leq 1$ this semigroup, properly normalized, converges to a limit which depends only on $a$.
\end{abstract}

1. Introduction. In this paper we investigate the integro-differential equation

$$
u_{t}+b u_{x}+c u=a \int_{0}^{a x} u(t, a x-y) \mu(d y),
$$

where $a$ and $b$ are positive constants, $c$ is a real number, $\mu$ is a finite Borel measure on the interval $[0, \infty)$, and $u:[0, \infty) \times[0, \infty) \rightarrow \mathbb{R}$ satisfies the initial-boundary condition

$$
\left\{\begin{array}{l}
u(0, x)=v(x), \\
u(t, 0)=0
\end{array}\right.
$$

Equation (1.1) has a probabilistic interpretation in the case when $c=$ $\mu([0, \infty))$. Namely, consider a particle moving with speed $b$ in the interval $[0, \infty)$. Assume that in every time interval $[t, t+\Delta t]$ the particle has the probability $c \Delta t+o(\Delta t)$ of changing its position from $x$ to $(x+\xi) / a$, where $\xi$ is a random variable with distribution $c^{-1} \mu$, i.e. $\operatorname{Prob}(\xi \in A)=c^{-1} \mu(\xi \in A)$. Denote by $u(t, x)$ the probability density function of the position of the particle at time $t$. Then (1.1) describes the evolution of $u(t, x)$ in time. If $a=1$ and $c=\mu([0, \infty))$ then (1.1) is known as the integro-differential Takacs equation and plays an important role in the theory of jump processes.

By means of a suitable substitution equation (1.1) may be converted into a special case with $b=c=1$ and $\mu([0, \infty))=1$. In this case (1.1) generates a semigroup of Markov operators on $L^{1}[0, \infty)$ given by $S^{t} v(x)=u(t, x)$. The asymptotic behaviour of this semigroup as $t \rightarrow \infty$ strongly depends on $a$.

1991 Mathematics Subject Classification: Primary 45K05. 
For $a>1$ this semigroup was studied by Klaczak [3]. He proved that if the measure $\mu$ is absolutely continuous with respect to the Lebesgue measure and $\int x \mu(d x)<\infty$, then the semigroup $\left\{S^{t}\right\}$ is asymptotically stable. In his proof he used the method of the lower bound function introduced by Lasota and Yorke [4] and developed by Dłotko and Lasota [1].

The main aim of this paper is to give the full description of the asymptotic properties of this semigroup. This description is given in Theorem 1 of Section 2. Sections 3 and 4 contain the proof of this theorem.

2. Main result. We denote by $D$ the set of all nonnegative elements of $L^{1}[0, \infty)$ with norm one. The elements of $D$ will be called densities. We will assume that $v \in D$. By setting $u(t, x)=0$ for $t \geq 0, x<0$ and $\mu(A)=0$ for $A \subset(-\infty, 0)$ equation (1.1) can be rewritten as

$$
u_{t}+b u_{x}+c u=P u(t, x),
$$

where $P: L^{1}(\mathbb{R}) \rightarrow L^{1}(\mathbb{R})$ is given by

$$
P f(x)=a \int f(a x-y) \mu(d y)=a(f * \mu)(a x) .
$$

From the Phillips perturbation theorem [2] equation (1.1) with the initialboundary condition (1.2) generates a semigroup $\left\{S^{t}\right\}$ of linear operators on $L^{1}(\mathbb{R})$ given by

$$
S^{t} v(x)=u(t, x)=e^{-c t} \sum_{n=0}^{\infty} T_{n}(t) v(x),
$$

where $T_{0}(t) v(x)=v(x-b t)$ and

$$
T_{n+1}(t) v(x)=\int_{0}^{t} T_{0}(t-s) P T_{n}(s) v(x) d s .
$$

It is easy to check that if $v(x)=0$ for $x<0$ then $P v(x)=0$ and $T_{0}(t) v(x)=$ 0 for $x<0$ and $t \geq 0$. Consequently, $S^{t} v(x)=0$ for $x<0$ and $t \geq 0$, which implies that $\left\{S^{t}\right\}$ is the semigroup generated by equation (1.1).

Now observe that substituting $\bar{u}(t, x)=e^{\lambda t} u(p t, r x)$ into (1.1), where $p=1 / d, r=b / d, \lambda=c / d-1$, and $d=\mu([0, \infty))$ we obtain

$$
\bar{u}_{t}+\bar{u}_{x}+\bar{u}=a \int \bar{u}(t, a x-y) \bar{\mu}(d y),
$$

where $\bar{\mu}$ is the probability measure on $[0, \infty)$ given by $\bar{\mu}(A)=\mu(r A) / d$. Since the properties of $u$ can easily be deduced from the properties of $\bar{u}$, in the remainder of this paper we assume that $b=c=1$ and $\mu([0, \infty))=1$. Let $u$ be the solution of (1.1) satisfying the initial condition $u(0, x)=v(x)$ and let $U(t, x)=\int_{0}^{x} u(t, y) d y$. Let $\Phi(x)=\int_{-\infty}^{x} \frac{1}{\sqrt{2 \pi}} e^{-y^{2} / 2} d y$ and $\varphi=\Phi^{\prime}$. 
Theorem 1. Assume that $v \in D$ and that $v$ is bounded.

(a) If $\int_{0}^{\infty} \ln (1+x) \mu(d x)<\infty$ and $a<1$, then $U\left(t, a^{-\sqrt{t} x-t}\right)$ converges uniformly to $\Phi(x)$ on $\mathbb{R}$ as $t \rightarrow \infty$.

(b) If $\int_{0}^{\infty} \ln (1+x) \mu(d x)<\infty$ and $a>1$, then there exists a continuous density $v_{0}$ independent of $v$ such that $u(t, x)$ converges uniformly to $v_{0}$ as $t \rightarrow \infty$. Moreover, $S^{t} v_{0}=v_{0}$ for $t>0$.

(c) If $a=1, m=\int x \mu(d x)<\infty, m>0$, and $k=\int x^{2} \mu(d x)<\infty$, then $U(t, \sqrt{k t} x+m t+t)$ converges uniformly to $\Phi(x)$. Moreover, if $\mu$ has a bounded density then $\sqrt{k t} u(t, \sqrt{k t} x+m t+t)$ converges uniformly to $\varphi(x)$ as $t \rightarrow \infty$.

R e m ark 1 . In the case $b=c=1$ and $\mu([0, \infty))=1,\left\{S^{t}\right\}$ is a semigroup of Markov operators, i.e. $S^{t} D \subset D$ for every $t>0$. From this and from Theorem 1(b), it follows immediately that if $a>1$ and $\int \ln (1+x) \mu(d x)<$ $\infty$, then for every $v \in D, S^{t} v \rightarrow v_{0}$ in $L^{1}$. This generalizes the result of Klaczak [3].

We divide the proof of Theorem 1 into a sequence of lemmas. In this section we give a formula for $T_{n}(t) v$.

LEMma 1. Let

$$
\varphi_{1}(t, x, a)= \begin{cases}\frac{a}{1-a} \mathbf{1}_{[t, t / a]}(x) & \text { for } a \in(0,1), \\ \frac{a}{a-1} \mathbf{1}_{[t / a, t]}(x) & \text { for } a>1 .\end{cases}
$$

Define

$$
\varphi_{n}(t, x, a)=\int_{0}^{t} a \varphi_{n-1}(s, a(x-t+s), a) d s
$$

for $t \geq 0, x \geq 0, a>0, a \neq 1$, and $n \geq 2$. Then for $n \geq 1$,

$$
T_{n}(t) v(x)=\left(\varphi_{n} * P^{n} v\right)(t, x)=\int_{-\infty}^{\infty} \varphi_{n}(t, y, a) P^{n} v(x-y) d y .
$$

Lemma 1 follows immediately from (2.4) and the definition of $P$. Using induction arguments it is easy to check the following lemma.

Lemma 2. Let $x_{+}=x$ if $x>0$ and $x_{+}=0$ if $x \leq 0$. Then for $n \geq 1$ we have

$$
\varphi_{n}(t, x, a)=\sum_{k=0}^{n} a_{n, k, a}\left(x-t a^{-k}\right)_{+}^{n-1}
$$

where

$$
a_{n, k, a}=\frac{(-1)^{k} a^{(n(n+1)+k(k-1)) / 2}}{(n-1) !(1-a) \ldots\left(1-a^{k}\right)(1-a) \ldots\left(1-a^{n-k}\right)}
$$


Corollary 1. For every $n \geq 1$ and $a>0, a \neq 1$,

$$
\varphi_{n}\left(t, x, a^{-1}\right)=a^{-n} \varphi_{n}\left(t, x a^{-n}, a\right) .
$$

3. Properties of $\varphi_{n}$. Although the functions $\varphi_{n}$ are given explicitly it is difficult to investigate their behaviour as $n \rightarrow \infty$ using only formula (2.8). Therefore we define, by induction, an auxiliary sequence of functions $\eta_{n}:[0, \infty) \rightarrow \mathbb{R}, n=1,2, \ldots$ Let $a \in(0,1), \eta_{1}(x)=(1-a)^{-1} \mathbf{1}_{[a, 1]}(x)$ and

$$
\eta_{n}(x)= \begin{cases}0 & \text { for } x \leq a^{n}, \\ n \int_{x}^{\infty} \frac{\left(x-a^{n}\right)^{n-1}}{\left(z-a^{n}\right)^{n}} \eta_{n-1}(z) d z & \text { for } x>a^{n} .\end{cases}
$$

LEMMA 3. For every $n \geq 1$,

$$
\varphi_{n}(t, x, a)=\frac{t^{n-1} a^{n}}{n !} \eta_{n}\left(\frac{x a^{n}}{t}\right) .
$$

Proof. Since $\varphi_{1}(t, x, a)=0$ for $x \leq t$, it follows from (2.6) that $\varphi_{n}(t, x, a)=0$ for $x \leq t, n>1$. For $n=1$ formula (3.2) is obvious. Assume that (3.2) holds for $n-1$. Then for $x>t$ we have

$$
\begin{aligned}
\varphi_{n}(t, x, a) & =\int_{0}^{t} a \varphi_{n-1}(s, a(x-t+s), a) d s \\
& =\int_{0}^{t} \frac{a^{n} s^{n-2}}{(n-1) !} \eta_{n-1}\left(\frac{(x-t+s) a^{n}}{s}\right) d s \\
& =\frac{a^{n} t^{n-1}}{n !} \int_{a^{n} x / t}^{\infty} \frac{n\left(a^{n} x / t-a^{n}\right)^{n-1}}{\left(z-a^{n}\right)^{n}} \eta_{n-1}(z) d z=\frac{a^{n} t^{n-1}}{n !} \eta_{n}\left(\frac{x a^{n}}{t}\right) .
\end{aligned}
$$

Now we give a probabilistic interpretation of $\left\{\eta_{n}\right\}$. Let $Y_{1}, Y_{2}, \ldots$ be a sequence of independent random variables such that

$$
h_{n}(x)=(n+1) x^{n} \mathbf{1}_{[0,1]}(x)
$$

is the density of $Y_{n}$.

LEMma 4. Let $X_{1}$ be a random variable independent of $Y_{1}, Y_{2}, \ldots$ and with density $\eta_{1}$. Then the random variables $X_{n}, n \geq 2$, defined inductively by

$$
X_{n}=\left(X_{n-1}-a^{n}\right) Y_{n-1}+a^{n},
$$

have densities $\eta_{n}$.

Proof. Since $\operatorname{supp} \eta_{1}=[a, 1]$ and $\operatorname{supp} h_{n}=[0,1]$, we may assume that $a \leq X_{1} \leq 1$ and $0 \leq Y_{n} \leq 1$. This implies that $a^{n} \leq X_{n} \leq 1$. Let 
$x \in\left(a^{n}, 1\right)$. Then

$$
\begin{aligned}
\operatorname{Prob}\left(X_{n}<x\right) & =\operatorname{Prob}\left(\left(X_{n-1}-a^{n}\right) Y_{n-1}+a^{n}<x\right) \\
& =\iint_{A} \eta_{n-1}(z) h_{n-1}(y) d y d z,
\end{aligned}
$$

where

$$
A=\left\{(y, z): a^{n-1} \leq z \leq 1,0 \leq y \leq 1,\left(z-a^{n}\right) y+a^{n}<x\right\} .
$$

Hence

$$
\operatorname{Prob}\left(X_{n}<x\right)=\int_{0}^{x} \int_{0}^{1} \eta_{n-1}(z) h_{n-1}(y) d y d z+\int_{x}^{\infty} \eta_{n-1}(z)\left(\frac{x-a^{n}}{z-a^{n}}\right)^{n} d z .
$$

This implies that the density of $X_{n}$ is given by (3.1).

LEMMA 5. Let

$$
g_{n}(x)=\frac{1}{n+1} \eta_{n}\left(\frac{x}{n+1}\right) .
$$

Then there exists a continuous density $g$ vanishing at $\infty$ such that $g_{n}$ converges uniformly to $g$ on $[0, \infty)$.

Proof. First we check that the sequence $\left\{g_{n}\right\}$ is relatively compact in the topology of uniform convergence on $[0, \infty)$. Indeed, from (3.1) it follows that

$$
\eta_{n}(x) \leq \int_{x}^{\infty} \frac{n\left(x-a^{n}\right)^{n-1}}{\left(z-a^{n}\right)^{n}} d z \sup \eta_{n-1} \leq \frac{n}{n-1} \sup \eta_{n-1} .
$$

This implies that

$$
\sup \eta_{n} \leq n \sup \eta_{1} .
$$

Integrating (3.1) by parts we obtain

$$
\eta_{n}(x)=\frac{n}{n-1} \eta_{n-1}(x)+\frac{n}{n-1}\left(x-a^{n}\right)^{n-1} \int_{x}^{\infty} \frac{\eta_{n-1}^{\prime}(z)}{\left(z-a^{n}\right)^{n-1}} d z .
$$

Consequently,

$$
\eta_{n}^{\prime}(x)=n\left(x-a^{n}\right)^{n-2} \int_{x}^{\infty} \frac{\eta_{n-1}^{\prime}(z)}{\left(z-a^{n}\right)^{n-1}} d z
$$

and

$$
\sup \left|\eta_{n}^{\prime}\right| \leq \frac{n}{n-2} \sup \left|\eta_{n-1}^{\prime}\right|
$$

This implies that

$$
\sup \left|\eta_{n}^{\prime}\right| \leq C n^{2}
$$


for $n=3,4, \ldots$, and some constant $C$. From the definition of $g_{n},(3.4)$ and (3.5) it follows that the sequences $\left\{g_{n}\right\}$ and $\left\{g_{n}^{\prime}\right\}$ are bounded. Let $X_{n}^{\prime}=(n+1) X_{n}$ and $Y_{n}^{\prime}=\frac{n+2}{n+1} Y_{n}$. Then $g_{n}$ is the density of $X_{n}^{\prime}$,

$$
E Y_{n}^{\prime}=1, \quad E X_{n}^{\prime 2}=1+\frac{1}{(n+1)(n+3)}, \quad Y_{n}^{\prime} \leq \frac{n+2}{n+1}
$$

and

$$
X_{n+1}^{\prime}=X_{n}^{\prime} Y_{n}^{\prime}-(n+1) a^{n+1} Y_{n}^{\prime}+(n+2) a^{n+1} .
$$

Since $X_{n}^{\prime}$ and $Y_{n}^{\prime}$ are independent, we have $E X_{n+1}^{\prime}=E X_{n}^{\prime}+a^{n+1}$ and, consequently,

$$
E X_{n}^{\prime}=1+a+\ldots+a^{n} \leq \frac{1}{1-a} .
$$

This and the Chebyshev inequality imply

$$
\operatorname{Prob}\left(X_{n}^{\prime} \geq M\right) \leq \frac{E X_{n}^{\prime}}{M} \leq \frac{1}{M(1-a)},
$$

which yields

$$
\int_{M}^{\infty} g_{n}(x) d x \leq \frac{1}{M(1-a)}
$$

Since $\left\{g_{n}^{\prime}\right\}$ is bounded, there exists a constant $K$ such that $g_{n}^{\prime}(x) \geq-K$ for $x \geq 0, n \geq 3$. Let $x_{0} \geq M$; then

$$
g_{n}(x) \geq g_{n}\left(x_{0}\right)-K\left(x-x_{0}\right) \quad \text { for } x \in\left[x_{0}, x_{0}+g_{n}\left(x_{0}\right) / K\right] .
$$

From this it follows that

$$
\int_{M}^{\infty} g_{n} d x \geq g_{n}^{2}\left(x_{0}\right) /(2 K) .
$$

Using (3.9) we obtain $g_{n}\left(x_{0}\right) \leq(2 K /((1-a) M))^{1 / 2}$ and, consequently,

$$
\lim _{M \rightarrow \infty} \sup _{n \geq 1} \sup _{x \geq M} g_{n}(x)=0 .
$$

Condition (3.10) and boundedness of $\left\{g_{n}\right\}$ and $\left\{g_{n}^{\prime}\right\}$ imply that $\left\{g_{n}\right\}$ is relatively compact. Moreover, from (3.9) it follows that all accumulation points of $\left\{g_{n}\right\}$ are densities. Now, we show that $\left\{g_{n}\right\}$ has only one accumulation point. Applying the inequality $Y_{n}^{\prime} \leq(n+2) /(n+1)$ to (3.6) we obtain $X_{n+1}^{\prime} \geq X_{n}^{\prime} Y_{n}^{\prime}$. Let

$$
Z_{n, k}=Y_{n}^{\prime} Y_{n+1}^{\prime} \ldots Y_{n+k-1}^{\prime} .
$$

Then $X_{n+k}^{\prime} \geq X_{n}^{\prime} Z_{n, k}$. Since $X_{n}^{\prime}, Y_{n}^{\prime}, \ldots, Y_{n+k-1}^{\prime}$ are independent and $E Z_{n, k}=1$, we have

$$
E\left(X_{n+k}^{\prime}-X_{n}^{\prime} Z_{n, k}\right)=E X_{n+k}^{\prime}-E X_{n}^{\prime} \leq \frac{a^{n+1}}{1-a} .
$$


Furthermore,

$$
E Z_{n, k}^{2} \leq \prod_{j=n}^{\infty}\left(1+\frac{1}{j^{2}}\right) .
$$

Thus $D^{2} Z_{n, k} \leq \beta(n)$, where $\lim _{n \rightarrow \infty} \beta(n)=0$. Let $\delta$ and $\varepsilon$ be fixed positive numbers and choose $n_{0}(\delta, \varepsilon)$ such that for $n \geq n_{0}(\delta, \varepsilon)$

$$
\beta(n) \leq \varepsilon^{2} \delta^{3}(1-a)^{2} / 108 \text { and } a^{n+1}<\varepsilon \delta(1-a) / 6 .
$$

Then from (3.8), (3.11) and the Chebyshev inequality it follows that

$$
\operatorname{Prob}\left(\left|X_{n+k}^{\prime}-X_{n}^{\prime} Z_{n, k}\right|>\varepsilon / 2\right) \leq 2 a^{n+1} /((1-a) \varepsilon)<\delta / 3,
$$

$$
\operatorname{Prob}\left(\left|Z_{n, k}-1\right|>\delta \varepsilon(1-a) / 6\right) \leq 36 D^{2} Z_{n, k} /\left(\delta^{2} \varepsilon^{2}(1-a)^{2}\right) \leq \delta / 3,
$$

and

$$
\operatorname{Prob}\left(X_{n}^{\prime}>3 /(\delta(1-a))\right) \leq \delta / 3 .
$$

The last three inequalities imply

$$
\operatorname{Prob}\left(\left|X_{n+k}^{\prime}-X_{n}^{\prime}\right| \leq \varepsilon\right)>1-\delta
$$

and, consequently, for every $\varepsilon>0$ we have

$$
\lim _{n \rightarrow \infty, m \rightarrow \infty} \operatorname{Prob}\left(\left|X_{m}^{\prime}-X_{n}^{\prime}\right|>\varepsilon\right)=0 .
$$

Hence $X_{n}^{\prime}$ converges in probability. It follows that $\left\{g_{n}\right\}$ has only one accumulation point $g$. Since $\left\{g_{n}\right\}$ is relatively compact, $g_{n}$ converges uniformly to $g$.

Rem ark 2. Since $g, g_{1}, g_{2}, \ldots$ are densities and $g_{n} \rightarrow g$ uniformly, $g_{n}$ converges to $g$ in $L^{1}$.

LEMMA 6. Let $g$ be the function from Lemma 5 corresponding to $a<1$. Then

$$
\int\left|\frac{n !}{t^{n}} \varphi_{n}(t, x, a)-a^{n} g\left(a^{n} x\right)\right| d x \rightarrow 0
$$

and

$$
\frac{n !}{t^{n}} \varphi_{n}\left(t, x, a^{-1}\right) \rightrightarrows g(x) \quad \text { on }[0, \infty)
$$

as $t \rightarrow \infty$ and $n / t \rightarrow 1$.

This follows immediately from Lemmas 3 and 5, Corollary 1 and Re$\operatorname{mark} 2$.

4. Convergence of solutions. We first examine the operator $P$. In this section we assume that $\mu$ satisfies

$$
\int_{0}^{\infty} \ln (1+x) \mu(d x)<\infty .
$$


Let $v \in D$ and let $\mu_{n, v}$ denote the Borel measure on $[0, \infty)$ defined by

$$
\mu_{n, v}(A)=\int_{A} P^{n} v d x .
$$

LEMma 7. (i) If $a>1$, then there exists a Borel probability measure $\mu_{0}$ such that for every $v \in D$ the measures $\mu_{n, v}$ converge weakly to $\mu_{0}$ as $n \rightarrow \infty$.

(ii) If $a<1$, then for every $v \in D$ there exists $v_{0} \in D$ such that the functions $v_{n}(x)=a^{-n} P^{n} v\left(a^{-n} x\right)$ converge in $L^{1}$ to $v_{0}$ as $n \rightarrow \infty$.

(iii) If $a=1, m=\int x \mu(d x)<\infty, k=\int x^{2} \mu(d x)<\infty, \sigma^{2}=k-m^{2}>$ $0, w_{n, v}(x)=\sqrt{n} \sigma P^{n} v(n m+x \sigma \sqrt{n})$ and $W_{n, v}(x)=\int_{-\infty}^{x} w_{n, v}(y) d y$ then $W_{n, v} \rightrightarrows \Phi$ on $\mathbb{R}$. Moreover, if $\mu$ has a bounded density then $w_{n, v} \rightrightarrows \varphi$ on $\mathbb{R}$.

The proof is partly based on the technique developed by Łoskot [5] who investigated iterates of random variables.

Proof. Let $\xi_{1}, \xi_{2}, \ldots$ be a sequence of independent random variables with distribution $\mu$, i.e. $\operatorname{Prob}\left(\xi_{i} \in A\right)=\mu(A)$, and let $X$ be a random variable independent of $\xi_{1}, \xi_{2}, \ldots$ with density $v$. Then $P^{n} v$ is the density of

$$
\zeta_{n}=a^{-n} X+a^{-1} \xi_{1}+\ldots+a^{-n} \xi_{n} .
$$

Let $a>1$. From the Kolmogorov three series theorem (see e.g. [7]) it follows that $\sum a^{-n} \xi_{n}$ converges a.e. if

$$
\sum_{n=1}^{\infty} E\left(\frac{a^{-n} \xi_{n}}{1+a^{-n} \xi_{n}}\right)<\infty .
$$

Since each $\xi_{n}$ has distribution $\mu$,

$$
\begin{aligned}
\sum_{n=1}^{\infty} E( & \left.\frac{a^{-n} \xi_{n}}{1+a^{-n} \xi_{n}}\right)=\sum_{n=1}^{\infty} \int_{0}^{\infty} \frac{a^{-n} x}{1+a^{-n} x} \mu(d x) \\
& <\int_{0}^{\infty} \int_{0}^{\infty} \frac{a^{-t} x}{1+a^{-t} x} \mu(d x) d t=\ln ^{-1} a \int_{0}^{\infty} \ln (1+x) \mu(d x)<\infty .
\end{aligned}
$$

This implies that $\zeta_{n}$ converges a.e. to some random variable $\zeta$ and, consequently, $\mu_{n, v}$ converges weakly to $\mu_{0}$, where $\mu_{0}(A)=\operatorname{Prob}(\zeta \in A)$.

Let $a<1$. Then $v_{n}$ is the density of $a^{n} \zeta_{n}$. Since the $\xi_{i}$ have the same distribution, from (4.3) it follows that $v_{n}$ is the density of $X+\xi_{1}+$ $\ldots+a^{n-1} \xi_{n}$. The series $\sum a^{n-1} \xi_{n}$ is a.e. convergent. This implies that $v_{n}$ converges in $L^{1}$ to some density.

Let $a=1$. That $W_{n, v} \rightrightarrows \Phi$ follows immediately from the central limit theorem. If $\mu$ has a bounded density, then we apply the local form of the central limit theorem (see e.g. [6]). 
Proof of Theorem 1. Let $N(t)$ be the Poisson process, i.e.

$$
\operatorname{Prob}(N(t)=n)=p_{n, t}=t^{n} e^{-n} / n ! .
$$

Let

$$
A_{t, \delta}=\{n \geq 0:|n-t|<\delta t\}, \quad \delta>0 .
$$

Since $E N(t)=t$ and $D^{2} N(t)=t$, the Chebyshev inequality implies

$$
\sum_{n \notin A_{t, \delta}} p_{n, t} \leq D^{2} N(t) /(\delta t)^{2}=1 /\left(\delta^{2} t\right) .
$$

Case 1: $a<1$. Let $\varepsilon>0$. Since $T_{n}(t)=\varphi_{n} * P^{n}$, Lemma 7 (ii) and (3.14) imply that for every $\varepsilon>0$ there exist $t_{0}>0$ and $\delta>0$ such that

$$
\int\left|t^{-n} n ! T_{n}(t) v(x)-a^{n}\left(v_{0} * g\right)\left(a^{n} x\right)\right| d x<\varepsilon
$$

for $t>t_{0}$ and $n \in A_{t, \delta}$. By the definition of $T_{n}(t) v, \int T_{n}(t) v=t^{n} / n$ !. Let

$$
w(t, x)=\sum_{n=0}^{\infty} p_{n, t} a^{n}\left(v_{0} * g\right)\left(a^{n} x\right) .
$$

From (4.4) and (4.5) it follows that

$$
\begin{aligned}
\int|u(t, x)-w(t, x)| d x & \leq \sum_{n=0}^{\infty} \int\left|e^{-t} T_{n}(t) v(x)-p_{n, t} a^{n}\left(v_{0} * g\right)\left(a^{n} x\right)\right| d x \\
& <\sum_{n \in A_{t, \delta}} \varepsilon p_{n, t}+\sum_{n \notin A_{t, \delta}} 2 p_{n, t}<\varepsilon+2 /\left(\delta^{2} t\right) .
\end{aligned}
$$

This implies that $u(t, \cdot)-w(t, \cdot)$ converges to 0 in $L^{1}$ as $t \rightarrow \infty$. Let

$$
W(t, x)=\int_{-\infty}^{x} w(t, y) d x, \quad F(x)=\int_{0}^{a^{-x}}\left(v_{0} * g\right)(y) d y
$$

and $H(t, x)=W\left(t, a^{-x}\right)$. Then $F$ is a distribution function and

$$
H(t, x)=\sum_{n=0}^{\infty} p_{n, t} F(x-n) .
$$

Let $X$ be a random variable independent of $N(t)$ with distribution function $F$. Then $H(t, x)$ are the distribution functions of the process $N(t)+X$. Since $(N(t)-t) / \sqrt{t}$ converges weakly to the normal distribution,

$$
H(t, t+x \sqrt{t}) \rightrightarrows \Phi(x) \quad \text { on } \mathbb{R} \text { as } t \rightarrow \infty,
$$

which gives

$$
U\left(t, a^{-\sqrt{t} x-t}\right) \rightrightarrows \Phi(x) \quad \text { on } \mathbb{R}
$$


Cas e 2: $a>1$. From Lemma 7(i) and (3.15) it follows that $t^{-n} n ! T_{n}(t) v$ converges uniformly to $g * \mu_{0}$ as $n \rightarrow \infty$ and $t \rightarrow \infty$ in such a way that $n / t \rightarrow 1$. Since $\left\{g_{n}\right\}$ is bounded, there exists $C>0$ such that

$$
\sup _{x} T_{n}(t) v \leq \sup _{x}\left|\varphi_{n}(t, x, a)\right| \leq t^{n-1} C /(n-1) ! .
$$

Now, using a similar argument to that in Case 1 we obtain $u(t, \cdot) \rightrightarrows g * \mu_{0}$ on $[0, \infty)$ as $t \rightarrow \infty$.

Case 3: $a=1$. It is easy to observe that the solution $u$ of (1.1) and (1.2) is given by

$$
u(t, x)=\sum_{n=0}^{\infty} p_{n, t} P^{n} v(x-t) .
$$

Let $k>m^{2}, G_{n}(x)=\int_{-\infty}^{x} P^{n} v(y) d y$ and

$$
Z(t, x)=U(t, \sqrt{k t} x+m t+t)=\sum_{n=0}^{\infty} p_{n, t} G_{n}(\sqrt{k t} x+m t) .
$$

Let $\varepsilon>0$. From Lemma 7 (iii) it follows that there exists $n_{0}>0$ such that

$$
\left|G_{n}(n m+y \sigma \sqrt{n})-\Phi(y)\right|<\varepsilon
$$

for $n \geq n_{0}$ and $y \in \mathbb{R}$. This implies that

$$
\left|G_{n}(\sqrt{k t} x+m t)-\Phi\left(\frac{\sqrt{k t} x+m(t-n)}{\sigma \sqrt{n}}\right)\right|<\varepsilon
$$

for $n \geq n_{0}$. Let $\delta>0$ be such that $|\Phi(x / \sqrt{n})-\Phi(x / \sqrt{t})|<\varepsilon$ for $n \in A_{t, \delta}$ and $x \in \mathbb{R}$. Then

$$
\left|G_{n}(\sqrt{k t} x+m t)-\Phi\left(\frac{\sqrt{k t} x+m(t-n)}{\sigma \sqrt{t}}\right)\right|<2 \varepsilon
$$

for $n \in A_{t, \delta}, x \in \mathbb{R}$ and sufficiently large $t$. Let

$$
W(t, x)=\sum_{n=0}^{\infty} p_{n, t} \Phi\left(\frac{\sqrt{k t} x+m(t-n)}{\sigma \sqrt{t}}\right) .
$$

Then

$$
|Z(t, x)-W(t, x)| \leq 2 \varepsilon+2 \sum_{n \notin A_{t, \delta}} p_{n, t} \leq 2 \varepsilon+2 /\left(\delta^{2} t\right)
$$

for sufficiently large $t$. This implies that

$$
\lim _{t \rightarrow \infty} \sup _{x \in \mathbb{R}}|Z(t, x)-W(t, x)|=0 .
$$

Similarly, if $v$ is a bounded function and $\mu$ has a bounded density then

$$
\lim _{t \rightarrow \infty} \sup _{x \in \mathbb{R}}|z(t, x)-w(t, x)|=0,
$$


where $z(t, x)=\frac{\partial Z}{\partial x}(t, x)$ and $w(t, x)=\frac{\partial W}{\partial x}(t, x)$. Now let $X$ be a random variable independent of the process $N(t)$ and with density $\varphi$. Then for every $t>0$ the function $w(t, x)$ is the density of the random variable

$$
Y(t)=\frac{\sigma X}{\sqrt{k}}+\frac{m(N(t)-t)}{\sqrt{k t}} .
$$

It is easy to check that the density of $Y(t)$ converges uniformly to $\varphi$ as $t \rightarrow \infty$, which completes the proof in the case $k>m^{2}$. If $k=m^{2}$ then $\mu$ is concentrated at $x=m$. This implies that

$$
u(t, x)=\sum_{n=0}^{\infty} p_{n, t} v(x-n m-t) .
$$

Now suppose that $\xi$ is a random variable independent of the process $N(t)$ and with density function $v$. Then $u(t, x)$ is the density of $X(t)=\xi+t+$ $m N(t)$. Since the distribution function of $(X(t)-t-m t) /(m \sqrt{t})$ converges uniformly to $\Phi$ as $t \rightarrow \infty$, we obtain

$$
U(t, m \sqrt{t} x+m t+t) \rightrightarrows \Phi(x) .
$$

\section{References}

[1] T. Dłotko and A. Lasota, Statistical stability and the lower bound function technique, in: Semigroups. Theory and Applications, Vol. I, H. Brezis, M. Crandall and F. Kappel (eds.), Longman Scientific \& Technical, 1987, 75-95.

[2] N. Dunford and J. T. Schwartz, Linear Operators, Part I, Interscience Publ., New York 1968.

[3] J. Klaczak, Stability of a transport equation, Ann. Polon. Math. 49 (1988), 69-80.

[4] A. Lasota and J. A. Yorke, Exact dynamical systems and the Frobenius-Perron operator, Trans. Amer. Math. Soc. 273 (1982), 375-384.

[5] K. Eoskot, Stochastic perturbations of dynamical systems, Ann. Polon. Math., to appear.

[6] A. Rényi, Probability Theory, Akadémiai Kiadó, Budapest 1970

[7] A. N. Shiryaev, Probability, Nauka, Moscow 1989 (in Russian).

INSTITUTE OF MATHEMATICS

SILESIAN UNIVERSITY

BANKOWA 14

40-007 KATOWICE, POLAND
INSTITUTE OF MATHEMATICS POLISH ACADEMY OF SCIENCES STAROMIEJSKA 8 40-013 KATOWICE, POLAND 